\title{
Lipoprotein(a) and proprotein convertase subtilisin/kexin type 9 inhibitors
}

\author{
U. Julius ${ }^{1} \cdot$ S. Tselmin ${ }^{1} \cdot$ U. Schatz ${ }^{1}$ S. Fischer ${ }^{1} \cdot$ S. R. Bornstein ${ }^{1}$
}

Published online: 5 March 2019

(c) The Author(s) 2019

\begin{abstract}
Lipoprotein(a) (Lp(a)) is an internationally accepted independent atherogenic risk factor. Details about its synthesis, many aspects of composition and clearance from the bloodstream are still unknown. LDL receptor (LDLR) (and probably other receptors) play a role in the elimination of $\mathrm{Lp}$ (a) particles. Proprotein convertase subtilisin/kexin type 9 (PCSK9) inhibitors increase the number of available LDLRs and in this way very effectively reduce the LDL cholesterol (LDL-C) concentrations. As shown in controlled studies using PCSK9 inhibitors, Lp(a) levels are decreased by 20 to 30\%, though in some patients no effect was observed. So far, it has not been clarified whether this decrease is associated with an effect on the incidence of cardiovascular events (CVEs). In two recently published well-performed secondary prevention studies (FOURIER with evolocumab, ODYSSEY OUTCOMES with alirocumab) baseline Lp(a) levels were shown to have an impact on CVEs independently of baseline LDL-C concentrations. The rather modest PCSK9 inhibitor-induced decrease of $\mathrm{Lp}$ (a) was associated with a reduction of CVEs in both studies, even after adjusting (ODYSSEY OUTCOMES) for demographic variables (age, sex, race, region), baseline Lp(a), baseline LDL-C, change in LDL-C, and clinical variables (time from acute coronary syndrome, body mass index, diabetes, smoking history). The largest decrease of CVEs was seen in patients with relatively low concentrations of both LDL-C and Lp(a) (FOURIER). These findings will probably have an influence on the use of PCSK9 inhibitors in patients with high Lp(a) concentrations.
\end{abstract}

Keywords Lipoprotein(a) - LDL cholesterol · PCSK9 inhibitors · Cardiovascular events $\cdot$ Lipoprotein apheresis

\section{Lipoprotein(a)-synthesis, composition, metabolism, and clinical significance}

Lipoprotein(a) (Lp(a)) consists of an LDL particle to which an apolipoprotein(a) (apo(a)) is linked with a single disulfide bond. The binding between apolipoprotein (B) (apoB), the major apolipoprotein of the LDL, and apo(a) takes place either in the hepatic cells, in the space of Disse, or in the vascular lumen [1]. The cholesterol content of the LDL in $\mathrm{Lp}$ (a) varies between 30 and $45 \%$. The $\mathrm{Lp}$ (a) concentration is genetically determined. Mutations in the Lp(a) gene (LPA) and especially a variable number of LPA kringles IV type 2 in the apo(a) have an effect. A low number of these kringles is associated with higher $\mathrm{Lp}(\mathrm{a})$ levels.

This article is part of the special issue "Lp(a) - Update 2018"

U. Julius

ulrich.julius@uniklinikum-dresden.de

1 Lipidology and Center for Extracorporeal Treatment, Department of Internal Medicine III, University Hospital Carl Gustav Carus, Technische Universität Dresden, Fetscherstr. 74, 01307 Dresden, Germany
The clearance of $\mathrm{Lp}(\mathrm{a})$ from the bloodstream is still not fully understood. Hepatic (LDL receptor (LDLR), VLDL receptor, scavenger receptor $\mathrm{B} 1$, LDL receptor-related protein 1, cluster of differentiation 36 receptor (CD36), plasminogen receptor) and nonhepatic receptors are probably involved [1-4]. Renal mechanisms may also play a role.

It is assumed that LDLRs only play a significant role in $\mathrm{Lp}$ (a) clearance when hepatic levels of the receptor are very high and LDL-C levels are low, as is the case in proprotein convertase subtilisin/kexin type 9 (PCSK9) inhibitor therapy. It is possible that apo(a) isoform length influences clearance behavior of $\mathrm{Lp}(\mathrm{a})$ in human plasma following upregulation of LDLRs.

The physiological significance of $L p(a)$ particles may relate to their procoagulatory properties. Thus, wound healing could be stimulated.

On the other hand, $\mathrm{Lp}$ (a) induces atherosclerotic lesions and is supposed to promote aortic valve stenosis: a combination of proatherosclerotic, proinflammatory and procoagulatory actions seems to be responsible [5]. Data suggest that the atherogenicity of $\mathrm{Lp}$ (a) may be mediated in part by proinflammatory oxidized phospholipids [6]. 
Plasma PCSK9 is found in association with Lp(a) particles in humans with high Lp(a) levels and in mice carrying human Lp(a) [7].

Studies using epidemiological data, Mendelian randomization and genome-wide associations have proven that elevated Lp(a) induce cardiovascular events (CVEs) like myocardial infarction (MI), stroke, occlusions of carotids or of leg arteries [5, 8].

New data obtained in the Danish population with respect to the relationship between $\operatorname{Lp}(a)$ and mortality have recently been published: In this study $\mathrm{Lp}$ (a) levels $>93 \mathrm{mg} / \mathrm{dl}$ (199 $\mathrm{nmol} / \mathrm{l}$; 96th-100th percentiles) versus $<10 \mathrm{mg} / \mathrm{dl}$ (18 nmol/1; 1st-50th percentiles) were associated with a hazard ratio (HR) of 1.50 (95\% CI 1.28-1.76) for cardiovascular mortality and of 1.20 (1.10-1.30) for all-cause mortality [9]. High levels of $\mathrm{Lp}(\mathrm{a})$, induced by low LPA kringle IV type 2 number of repeats rather than through high cholesterol content, were associated with increased mortality.

$\mathrm{Lp}$ (a) levels in the atherothrombotic range are generally accepted as $>30$ to $50 \mathrm{mg} / \mathrm{dl}$ or $>75$ to $125 \mathrm{nmol} / \mathrm{l}$ [1]. Such levels affect 20 to $30 \%$ of the global population, with possibly higher incidence in patients with established cardiovascular disease and calcific aortic valve disease.

\section{PCSK9 inhibitors-mode of action}

PCSK9 inhibitors are human monoclonal antibodies binding to the PCSK9 protein. This protein binds to LDLRs for endocytosis and lysosome degradation in the liver, resulting in an increase in circulating LDL cholesterol (LDL-C) level. LDLRs usually recycle after they transported LDL particles into the cells. By inhibiting this LDLR destruction, the number of LDLRs at the cell surface markedly increases-leading to an effective removal of circulating LDL particles. Thus, reductions of LDL-C of more than $50 \%$ can be reached.

Evidently, the PCSK9 protein also exerts effects on other receptors like VLDL receptor, LDL receptor-related protein 1 or the apolipoprotein E receptor [3].

It was shown that PCSK9 may increase the secretion of apo(a) and of apoB-an inhibition of PCSK9 may counteract these effects [10]. Thus PCSK9 inhibitors reduce Lp(a) levels by both increasing clearance and reducing its synthesis.

Lp(a) kinetics were studied using intravenous D3-leucine administration, mass spectrometry, and compartmental modeling [11]. Evolocumab monotherapy was shown to lower the plasma $\mathrm{Lp}(\mathrm{a})$ pool size by decreasing the production of $\mathrm{Lp}$ (a) particles. In combination with atorvastatin, evolocumab lowered the plasma Lp(a) pool size by accelerating the catabolism of $\mathrm{Lp}(\mathrm{a})$ particles.

\section{Effects of PCSK9 inhibitors on Lp(a) concentrations}

\section{Meta-analysis of randomized controlled trials with PCSK9 inhibitors}

A meta-analysis of studies using PCSK9 inhibitors (27 randomized controlled trials [RCTs] in 11,864 patients) documented a mean reduction of $\mathrm{Lp}$ (a) levels by $21.90 \%$ (95\% CI 24.28, 19.51) [10]. Treatment modalities like type of PCSK9 inhibitor, duration of therapy, application in patients with or without familial hypercholesterolemia, monotherapy or combination therapy, types of control treatment (placebo, ezetimibe), baseline Lp(a) (below 50 or above $50 \mathrm{mg} / \mathrm{dl}$ ), and immunoassay did not have a significant influence on this reduction. The lower the reached LDL-C concentration was, the higher the reduction rate for $\mathrm{Lp}(\mathrm{a})$. Attention should be paid to the fact that in the majority of the included studies mean $\mathrm{Lp}$ (a) ranged between 9 and $40 \mathrm{mg} / \mathrm{dl}$; however, in one investigation this value was 100 (but SD 162!) mg/dl.

\section{Evolocumab in patients with very high Lp(a) concentrations}

In 65 patients with $\mathrm{Lp}$ (a) concentrations of about $200 \mathrm{nmol} / \mathrm{l}$ an injection therapy with $420 \mathrm{mg}$ evolocumab once in 4 weeks reduced $\mathrm{Lp}$ (a) after 16 weeks by $28.0(56.5,9.0)$ nmol/l (median, IQR), equivalent to $13.9(19.3,8.5)$ percent (mean, 95\% CI) [12]. LDL-C was decreased by $2.2(0.8)$ $\mathrm{mmol} / \mathrm{l}$, corresponding to $60.7(65.8,55.5)$ percent (mean, $95 \% \mathrm{CI})$.

Interestingly, arterial wall inflammation (most diseased segment target-to-background ratio (MDS TBR)) in the index vessel (left carotid, right carotid, or thoracic aorta) was not changed by evolocumab. The explanation of the authors for this lack of change is that $\mathrm{Lp}$ (a) levels remained high despite the rather modest reduction induced by the PCSK9 inhibitor.

\section{Effect of PCSK9 inhibitors in patients undergoing lipoprotein apheresis therapy}

\section{ODYSSEY ESCAPE Study}

Alirocumab (150 mg biweekly, 2:1 allocation to verum and placebo, respectively) was given to patients $(n=62)$ who were treated with lipoprotein apheresis (LA) in order to evaluate the possibility to replace LA therapy by the PCSK9 inhibitor [13]. Alirocumab reduced $L p(a)$ levels in those patients with normal values (mean below $20 \mathrm{mg} / \mathrm{dl}$ ) after 6 weeks by $15 \%$ (placebo controlled) and after 18 weeks by $2.7 \%$. But in patients with high baseline $\mathrm{Lp}(\mathrm{a})$ concen- 

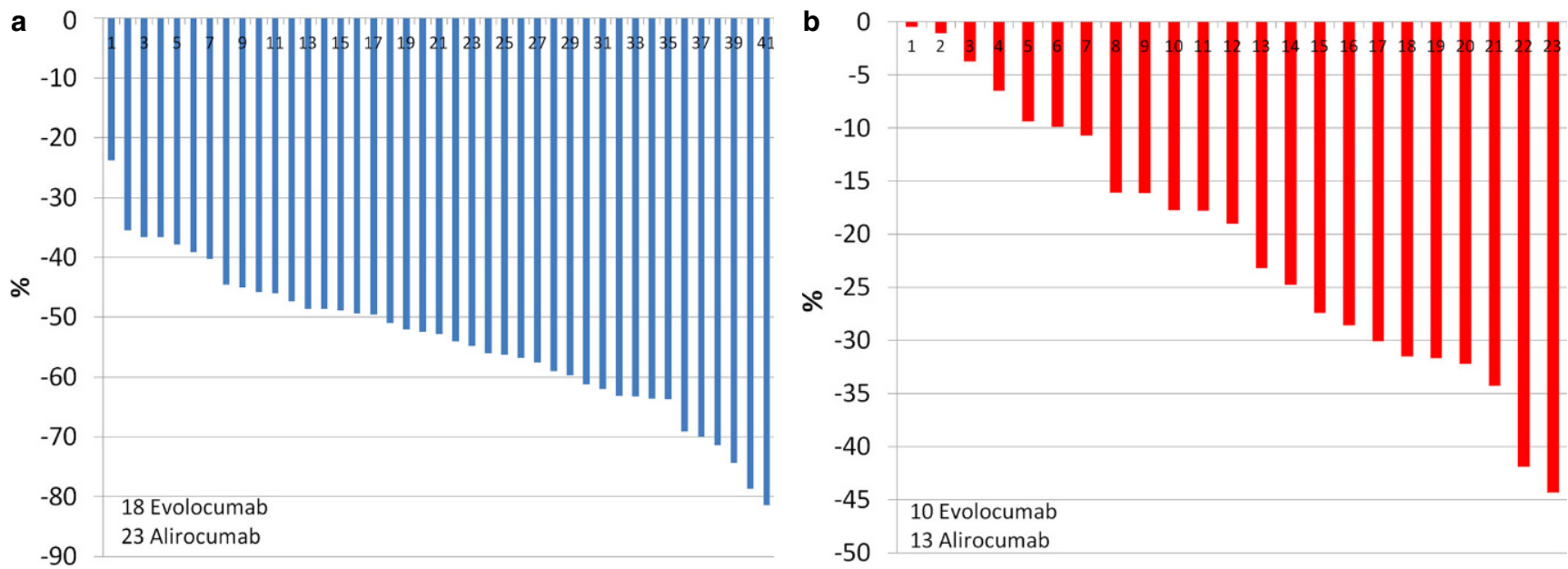

\begin{tabular}{|c|c|c|c|}
\hline & LDL-C 1 $(\mathrm{mmol} / \mathrm{l})$ & LDL-C 2 (mmol/l) & LDL-C reduction \\
\hline $\mathrm{m}$ & 3.66 & 1.74 & $-53.85 \%$ \\
\hline $\mathrm{sd}$ & 1.24 & 0.87 & 0.12 \\
\hline Max & 8.24 & 4.16 & $-23.79 \%$ \\
\hline Min & 1.48 & 0.38 & $-81.42 \%$ \\
\hline
\end{tabular}

\begin{tabular}{|c|c|c|c|}
\hline & Lp(a) 1 (nmol/I) & Lp(a) 2 (nmol/I) & Lp(a) reduction \\
\hline mean & 199.00 & 156.35 & $-20.80 \%$ \\
\hline SD & 74.29 & 55.49 & 0.13 \\
\hline Max & 376.00 & 247.00 & $-0.46 \%$ \\
\hline Min & 90.00 & 61.00 & $-44.35 \%$ \\
\hline
\end{tabular}

Fig. 1 Individual percent reductions of LDL-C (a, $n=41)$ and of Lp(a) (b, $n=23)$ after 12 weeks of PCSK9 inhibitor application (usually biweekly) in patients on LA therapy. $L D L-C$ LDL cholesterol, $L p(a)$ Lipoprotein(a)

trations (mean above $90 \mathrm{mg} / \mathrm{dl}$ ) the corresponding changes amounted to $13 \%$ and to $+1.9 \%$ (placebo controlled).

\section{Observations of the authors}

At our center we initiated PCSK9 inhibitor therapy in patients undergoing LA treatment when LDL-C levels remained high despite maximally tolerated lipid-lowering therapy (drugs, LA). Fig. 1 shows clear differences between patients with respect to lowering of LDL-C and $\mathrm{Lp}$ (a) (12 weeks after start of the injection therapy), indicating that reporting mean values is of limited significance when describing the effectiveness of PCSK9 inhibitors.

Among the 41 patients who started PCSK9 inhibitor therapy, only 23 showed elevated Lp(a) levels (higher than $120 \mathrm{nmol} / \mathrm{l}$ before first LA session).

\section{Outcome data in prospective controlled PCSK9 inhibitor studies-association with the effects of these drugs on Lp(a) levels}

\section{FOURIER Study}

The randomized FOURIER (Further Cardiovascular Outcomes Research with PCSK9 Inhibition in Patients with Elevated Risk) Study tested the effect of evolocumab on cardiovascular outcomes versus placebo in patients with established atherosclerotic cardiovascular disease (median follow-up 2.2 years) [14].
Lp(a) was measured in 25,096 patients [15]. The median (IQR) baseline $\mathrm{Lp}$ (a) concentration was $37(13,165)$ nmol/l. In the placebo arm, patients with baseline $L p(a)$ in the highest quartile had a higher risk of coronary heart disease (CHD) death, MI or urgent revascularization (UR) (adjusted HR Q4:Q1 1.22, 95\% CI 1.01,1.48) independent of LDL-C. At 48 weeks, evolocumab significantly reduced $\mathrm{Lp}(\mathrm{a})$ by a median (IQR) of $26.9 \%(6.2,46.7 \%)$ - equivalent to $11 \mathrm{nmol} / 1(1,32)$ absolute change. It is important to note that in more than $30 \%$ of the patients receiving evolocumab no reduction of $L p(a)$ was seen!

The percent change in $\mathrm{Lp}$ (a) and LDL-C at 48 weeks in evolocumab patients was moderately positively correlated. Evolocumab reduced the risk of CHD death, MI or UR by $23 \%$ (HR $0.77,95 \%$ CI $0.67,0.88$ ) in patients with a baseline $\mathrm{Lp}(\mathrm{a})>$ median, and by $7 \%$ (HR $0.93,0.80,1.08$ ) in those $\leq$ median. Coupled with the higher baseline risk, the absolute risk reductions and number-needed-to-treat for 3 years (NNT3y) were $2.49 \%$ and 40 vs. $0.95 \%$ and 105 , respectively.

When a clinical threshold of $120 \mathrm{nmol} / 1(50 \mathrm{mg} / \mathrm{dl})$ was applied, the absolute risk reductions and NNT3y were $2.41 \%$ and 41 for those above the threshold versus $1.41 \%$ and 71 below the threshold.

In a weighted least square linear regression analysis that examined the association between treatment effect on CHD death, MI or UR and per unit decrease in Lp(a) adjusting for differences in LDL-C, there was a significant relationship with a $15 \%$ relative risk reduction $(95 \%$ CI $2,26 \%$, $P=0.0199)$ per $25 \mathrm{nmol} / 1$ reduction in $\mathrm{Lp}(\mathrm{a})$. 
Table 1 CHD death, MI and UR beyond week 12 in dependence on reached Lp(a) (median $29 \mathrm{nmol} / \mathrm{l}$ ) and LDL-C (median $65 \mathrm{mg} / \mathrm{dl}$ ) levels

\begin{tabular}{|c|c|c|}
\hline & $\begin{array}{l}\text { LDL-C }>\text { median } \\
(\%)\end{array}$ & $\begin{array}{l}\text { LDL-C } \leq \text { median } \\
(\%)\end{array}$ \\
\hline $\mathrm{Lp}(\mathrm{a})>$ median & 8.52 & 7.09 \\
\hline $\mathrm{Lp}(\mathrm{a}) \leq$ median & 7.33 & 5.86 \\
\hline
\end{tabular}

$L D L-C$ LDL cholesterol, $\operatorname{Lp}($ a) Lipoprotein(a)

The authors observed a stepwise decrease in the risk of CHD death, MI or UR for patients who achieved either an Lp(a) or LDL-C value below the achieved median with the lowest event rate observed for those who achieved lower levels of both values. Compared with patients above the median achieved level for both lipid parameters, patients with at least one level below the median had a $15 \%$ lower risk of major coronary events (adjusted HR 0.85, 95\% CI 0.75, $0.97, P=0.01)$ and those with both levels below their respective medians had a $29 \%$ lower risk of major coronary events (adjusted HR 0.72, 95\% CI 0.62, 0.83, $P<0.0001$ ). It was reported that consistent results were observed when patients were stratified by achieved values of LDL-C $70 \mathrm{mg} / \mathrm{dl}$ and $L p$ (a) $120 \mathrm{nmol} / 1$.

The incidence (3y KMrate, \%) of the endpoint CHD death, MI and UR beyond week 12 was different depending on whether the reached Lp(a) and LDL-C levels where above or below the median (Table 1; [15]).

\section{ODYSSEY OUTCOMES Trial}

This is a multicenter, randomized, double-blind, placebocontrolled trial involving 18,924 patients who had an acute coronary syndrome 1 to 12 months earlier, had an LDL-C of at least $1.8 \mathrm{mmol} / \mathrm{l}(70 \mathrm{mg} / \mathrm{dl})$, a non-high-density lipoprotein cholesterol level of at least $2.6 \mathrm{mmol} / \mathrm{l}(100 \mathrm{mg} / \mathrm{dl})$, or an apoB level of at least $80 \mathrm{mg} / \mathrm{dl}$, and were receiving statin therapy at a high-intensity dose or at the maximum tolerated dose [16]. Patients were randomly assigned to receive alirocumab subcutaneously or matching placebo every 2 weeks.

Data on $\mathrm{Lp}$ (a) have been presented at the ISA congress in Toronto (2018). The median level was $21.2 \mathrm{mg} / \mathrm{dl}$ (IQR 6.7, 59.6). Major CVEs occurred in the baseline 4th quartile more often than in the 1st quartile (HR unadjusted 1.37; adjusted (for age, sex, race, geographic region, time since event, BMI, smoking history, diabetes, baseline LDL-C) 1.28). A similar relationship was observed for non-fatal MI. No relationship was found with stroke, cardiovascular death, or all-cause death. In the course of the study alirocumab reduced $\mathrm{Lp}(\mathrm{a})$ levels in the mean by about $5 \mathrm{mg} / \mathrm{dl}$. After 4 months these reductions amounted to $9.8 \mathrm{mg} / \mathrm{dl}$ (median; IQR $3.18,16.2$ ) in the 3rd quartile and to $20.2 \mathrm{mg} / \mathrm{dl}$ in the 4 th quartile (median; IQR $8.0,34.3$ ), more than in the 1 st and 2 nd quartiles.
Major CVEs were significantly reduced by alirocumab in the baseline 3rd (HR 0.79 (95\% CI 0.66, 0.95)) and 4th quartiles (HR 0.83 (95\% CI 0.70, 0.98)). Similar HRs were obtained after adjusting as detailed above. When the time-weighted moving average $\mathrm{Lp}$ (a) change from baseline was modeled with major CVEs or non-fatal MI significant reductions of events (approximately by 16\%) were seen, even after adjusting for demographic variables (age, sex, race, region), baseline $\mathrm{Lp}(\mathrm{a})$, baseline LDL-C, change in LDL-C, and clinical variables (time from acute coronary syndrome, body mass index, diabetes, smoking history).

\section{Conclusions}

An elevation of $L p(a)$ is currently no accepted indication for PCSK9 inhibitors. Two reasons explain this situation: (1) comparing with the effect on LDL-C concentrations, the decrease of Lp(a) under PCSK9 inhibitors is rather small - even absent in many patients, and (2) the association of the described reduction of $L p$ (a) levels by $20-30 \%$ with CVEs was unknown-new data on this topic appeared only recently.

In two prospective controlled intervention studies with both available PCSK9 inhibitors (evolocumab, alirocumab) it could be shown that elevated baseline Lp(a) levels represent an atherogenic risk factor, independently of baseline LDL-C concentrations.

It has to be remembered that $L p(a)$ levels were no inclusion criterion. Nonetheless, in the FOURIER Study approximately $33.1 \%$ of patients had a baseline concentration higher than $120 \mathrm{nmol} / 1$ (or approximately $50 \mathrm{mg} / \mathrm{dl}$ ) which is believed to be the 80th percentile in a general patient population [15].

The lowering of $L p(a)$ with the injection therapy reduced the rate of CVEs-with modeling an influence of baseline values or PCSK9 inhibitor-induced changes of LDL$\mathrm{C}$ or other factors could be excluded. The higher the baseline $L p(a)$ concentrations were, the higher the reduction of CVEs by the PCSK9 inhibitors was. These findings clearly put this new class of lipid-lowering drugs into another perspective. Possibly high Lp(a) levels will be taken into consideration when considering the use of these drugs in the future. Of course, this indication would be valid only in patients whose $L p(a)$ levels really demonstrate a decrease on this injection therapy.

The missing effect of PCSK9 inhibitors on Lp(a) concentrations in up to $30 \%$ of patients is not yet fully understood. Reasons, discussed in the literature, are the following: (1) apo(a) with a low kringle IV type 2 number may less actively bind to the LDLR, and (2) because furin-cleaved PCSK9 is somewhat less effective on binding to LDLRs compared with the intact PCSK9 form, it is possible that 
the balance between forms, as influenced by treatment with a PCSK9 inhibitor, also contributes to the degree of $\mathrm{Lp}$ (a) reduction on therapy [1].

The current therapeutic approach to improve the highrisk situation in patients with high $\mathrm{Lp}(\mathrm{a})$ levels is to optimize LDL-C below $1.8 \mathrm{mmol} / \mathrm{l}(70 \mathrm{mg} / \mathrm{dl})$.

Given the proposed potentiation of the CVD risk between LDL-C and Lp(a), Verbeek et al. hypothesized in 2018 that the risk associated with elevated $\mathrm{Lp}(\mathrm{a})$ levels would largely be attenuated at lower LDL-C levels [17]. They tested this hypothesis in two large studies corresponding to a primary prevention setting: the European Prospective Investigation of Cancer (EPIC) Norfolk prospective population study and the Copenhagen City Heart Study prospective population study. At LDL-C levels, corrected for Lp(a)-derived LDL-C, less than $2.5 \mathrm{mmol} / \mathrm{l}(\sim 100 \mathrm{mg} / \mathrm{dl})$, the risk associated with elevated $\mathrm{Lp}(\mathrm{a})$ decreases [17].

On the other hand, in statin studies where usually patients within secondary prevention were included, this situation is different. In an individual-patient data meta-analysis of statin-treated patients, patient-level data from seven randomized, placebo-controlled, statin outcomes trials were collated and harmonized to calculate HRs for CVEs, defined as fatal or non-fatal coronary heart disease, stroke, or revascularization procedures [18]. Elevated baseline and onstatin $L p(a)$ showed an independent approximately linear relation with cardiovascular disease risk, evident on treatment with either statin or placebo.

Statins in contrast to PCSK9 inhibitors do not decrease $\mathrm{Lp}$ (a) levels, but may even increase them. The parallel effect on both LDL-C and Lp(a) concentrations, as described in PCSK9 inhibitor studies, appears to be a major progress. It could be clearly shown that the optimization of both parameters reduces risk, but even the additional decrease of LDL-C represents an advantage.

The measured LDL-C also contains cholesterol that is transported with the Lp(a) particles [19]. This is a problem in patients who on PCSK9 inhibitor therapy achieved rather low LDL-C levels and who still show high $\mathrm{Lp}$ (a) values.

However, some patients still develop CVEs although both target levels were rather low during the course of the studies. The life-long burden with these lipoproteins could be a possible explanation; both LDL-C and Lp(a) are genetically determined.

In this context, the authors wish to emphasize that the rate of new events in high-risk patients with elevated $\mathrm{Lp}(\mathrm{a})$ concentrations on LA therapy is much lower $[20,21]$.

In the future, an antisense oligonucleotide against $L p(a)$ will be tested in a phase III study which will probably start in 2020. This study will clarify the effect of a of Lp(a) reduction by more than $90 \%$ on CVEs [22].
Conflict of interest U. Julius: honoraria from Aegerion, Akcea, Amgen, Amryt, Chiesi, Sanofi, Kaneka, Diamed, Fresenius Medical Care, MSD. S. Tselmin received honoraria for lectures and consulting by Amgen, Fresenius Medical Care, Kaneka, MSD, and Sanofi-Aventis. S. Fischer: honoraria from Sanofi, Amgen, MSD, Berlin-Chemie, Abbott, Boehringer Ingelheim. U. Schatz and S. R. Bornstein declare that they have no competing interests.

Open Access This article is distributed under the terms of the Creative Commons Attribution 4.0 International License (http:// creativecommons.org/licenses/by/4.0/), which permits unrestricted use, distribution, and reproduction in any medium, provided you give appropriate credit to the original author(s) and the source, provide a link to the Creative Commons license, and indicate if changes were made.

\section{References}

1. Tsimikas S, Fazio S, Ferdinand KC, Ginsberg HN, Koschinsky ML, Marcovina SM et al (2018) NHLBI working group recommendations to reduce lipoprotein(a)-mediated risk of cardiovascular disease and aortic stenosis. J Am Coll Cardiol 71(2):177-192. https:// doi.org/10.1016/j.jacc.2017.11.014

2. Sharma M, Redpath GM, Williams MJ, McCormick SP (2017) Recycling of apolipoprotein(a) after PlgRKT-mediated endocytosis of lipoprotein(a). Circ Res 120(7):1091-1102. https://doi.org/10. 1161/CIRCRESAHA.116.310272

3. Schulz R, Schluter KD (2017) PCSK9 targets important for lipid metabolism. Clin Res Cardiol Suppl. https://doi.org/10.1007/ s11789-017-0085-0

4. Reyes-Soffer G, Pavlyha M, Ngai C, Thomas T, Holleran S, Ramakrishnan R et al (2017) Effects of PCSK9 inhibition with alirocumab on lipoprotein metabolism in healthy humans. Circulation 135(4):352-362. https://doi.org/10.1161/CIRCULATIONAHA. 116.025253

5. Tsimikas S (2017) A test in context: lipoprotein(a): diagnosis, prognosis, controversies, and emerging therapies. J Am Coll Cardiol 69(6):692-711. https://doi.org/10.1016/j.jacc.2016.11.042

6. Tsimikas S, Brilakis ES, Miller ER, McConnell JP, Lennon RJ, Kornman KS et al (2005) Oxidized phospholipids, Lp(a) lipoprotein, and coronary artery disease. N Engl J Med 353(1):46-57. https:// doi.org/10.1056/NEJMoa043175

7. Tavori H, Christian D, Minnier J, Plubell D, Shapiro MD, Yeang $\mathrm{C}$ et al (2016) PCSK9 association with lipoprotein(a). Circ Res 119(1):29-35. https://doi.org/10.1161/CIRCRESAHA.116.308811

8. Nave AH, Lange KS, Leonards CO, Siegerink B, Doehner W, Landmesser U et al (2015) Lipoprotein (a) as a risk factor for ischemic stroke: a meta-analysis. Atherosclerosis 242(2):496-503

9. Langsted A, Kamstrup PR, Nordestgaard BG (2019) High lipoprotein(a) and high risk of mortality. Eur Heart J. https://doi.org/10. 1093/eurheartj/ehy902

10. Cao YX, Liu HH, Li S, Li JJ (2018) A Meta-analysis of the effect of PCSK9-monoclonal antibodies on circulating lipoprotein (a) levels. Am J Cardiovasc Drugs. https://doi.org/10.1007/s40256-0180303-2

11. Watts GF, Chan DC, Somaratne R, Wasserman SM, Scott R, Marcovina SM et al (2018) Controlled study of the effect of proprotein convertase subtilisin-kexin type 9 inhibition with evolocumab on lipoprotein(a) particle kinetics. Eur Heart J 39(27):2577-2585. https://doi.org/10.1093/eurheartj/ehy122

12. Stiekema LCA, Stroes ESG, Verweij SL, Kassahun H, Chen L, Wasserman SM et al (2018) Persistent arterial wall inflammation in patients with elevated lipoprotein(a) despite strong low-density lipoprotein cholesterol reduction by proprotein convertase subtil- 
isin/kexin type 9 antibody treatment. Eur Heart J. https://doi.org/10. 1093/eurheartj/ehy862

13. Moriarty PM, Parhofer KG, Babirak SP, Cornier MA, Duell PB, Hohenstein B et al (2016) Alirocumab in patients with heterozygous familial hypercholesterolaemia undergoing lipoprotein apheresis: the ODYSSEY ESCAPE trial. Eur Heart J 37(48): 3588-3595. https://doi.org/10.1093/eurheartj/ehw388

14. Giugliano RP, Pedersen TR, Park JG, De Ferrari GM, Gaciong ZA, Ceska R et al (2017) Clinical efficacy and safety of achieving very low LDL-cholesterol concentrations with the PCSK9 inhibitor evolocumab: a prespecified secondary analysis of the FOURIER trial. Lancet 390(10106):1962-1971. https://doi.org/10. 1016/S0140-6736(17)32290-0

15. O’Donoghue ML, Fazio S, Giugliano RP, Stroes ESG, Kanevsky E, Gouni-Berthold I et al (2018) Lipoprotein(a), PCSK9 inhibition and cardiovascular risk: insights from the FOURIER trial. Circulation. https://doi.org/10.1161/CIRCULATIONAHA.118.037184

16. Schwartz GG, Steg PG, Szarek M, Bhatt DL, Bittner VA, Diaz R et al (2018) Alirocumab and cardiovascular outcomes after acute coronary syndrome. N Engl J Med 379(22):2097-2107. https://doi. org/10.1056/NEJMoa1801174

17. Verbeek R, Hoogeveen RM, Langsted A, Stiekema LCA, Verweij SL, Hovingh GK et al (2018) Cardiovascular disease risk associated with elevated lipoprotein(a) attenuates at low low-density lipoprotein cholesterol levels in a primary prevention setting. Eur Heart J 39(27):2589-2596. https://doi.org/10.1093/eurheartj/ehy334
18. Willeit P, Ridker PM, Nestel PJ, Simes J, Tonkin AM, Pedersen TR et al (2018) Baseline and on-statin treatment lipoprotein(a) levels for prediction of cardiovascular events: individual patient-data meta-analysis of statin outcome trials. Lancet 392(10155):1311-1320. https://doi.org/10.1016/S0140-6736(18) 31652-0

19. Yeang C, Witztum JL, Tsimikas S (2015) 'LDL-C' = LDL-C + $\mathrm{Lp}(\mathrm{a})-\mathrm{C}$ : implications of achieved ultra-low LDL-C levels in the proprotein convertase subtilisin/kexin type 9 era of potent LDL-C lowering. Curr Opin Lipidol 26(3):169-178

20. Leebmann J, Roeseler E, Julius U, Heigl F, Spitthoever R, Heutling D et al (2013) Lipoprotein apheresis in patients with maximally tolerated lipid-lowering therapy, lipoprotein(a)-hyperlipoproteinemia, and progressive cardiovascular disease: prospective observational multicenter study. Circulation 128(24):2567-2576. https://doi.org/ 10.1161/CIRCULATIONAHA. 113.002432

21. Roeseler E, Julius U, Heigl F, Spitthoever R, Heutling D, Breitenberger P et al (2016) Lipoprotein apheresis for lipoprotein(a)-associated cardiovascular disease: prospective 5 years of follow-up and apolipoprotein(a) characterization. Arterioscler Thromb Vasc Biol 36(9):2019-2027. https://doi.org/10.1161/ATVBAHA.116.307983

22. Viney NJ, van Capelleveen JC, Geary RS, Xia S, Tami JA, Yu RZ et al (2016) Antisense oligonucleotides targeting apolipoprotein(a) in people with raised lipoprotein(a): two randomised, doubleblind, placebo-controlled, dose-ranging trials. Lancet 388(10057): 2239-2253. https://doi.org/10.1016/S0140-6736(16)31009-1 